\title{
Physics beyond the standard model with BlackHawk v2.0
}

\author{
Alexandre Arbey ${ }^{1,2,3, a}$, Jérémy Auffinger ${ }^{1, b}{ }_{(\mathbb{C}}$ \\ ${ }^{1}$ Univ Lyon, Univ Claude Bernard Lyon 1, CNRS/IN2P3, IP2I Lyon, UMR 5822, F-69622 Villeurbanne, France \\ 2 Theoretical Physics Department, CERN, 1211 Geneva 23, Switzerland \\ ${ }^{3}$ Institut Universitaire de France (IUF), 103 boulevard Saint-Michel, 75005 Paris, France
}

Received: 1 September 2021 / Accepted: 28 September 2021 / Published online: 16 October 2021

(C) The Author(s) 2021

\begin{abstract}
We present the new version $\mathrm{v} 2.0$ of the public code BlackHawk designed to compute the Hawking radiation of black holes, with both primary and hadronized spectra. This new version aims at opening an avenue toward physics beyond the Standard Model (BSM) in Hawking radiation. Several major additions have been made since version v1. 0: dark matter/dark radiation emission, spin 3/2 greybody factors, scripts for cosmological studies, BSM black hole metrics with their associated greybody factors and a careful treatment of the low energy showering of secondary particles; as well as bug corrections. We present, in each case, examples of the new capabilities of BlackHawk.
\end{abstract}

\section{Contents}

1 Introduction . . . . . . . . . . . . 1

2 New features ........................ 2

2.1 Adding a DM particle . . . . . . . . . . 2

2.2 Spin $3 / 2$ particle . . . . . . . . . . . 3

2.3 Time dependent features, cosmological studies . 4

2.4 New BH metrics and greybody factors . . . . . 5

2.5 Low energy photon spectrum . . . . . . . . 7

3 Parameters ................. . . 8

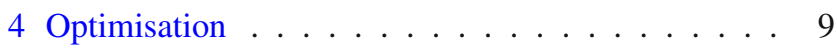

5 Conclusion . . . . . . . . . . . . . . 10

Appendix A: Routines . . . . . . . . . . . . 10

1. Modified routines . . . . . . . . . . . 10

2. New routines . . . . . . . . . . . . . . 11

Appendix B: Corrections . . . . . . . . . . . . . . . . . . 12

References . . . . . . . . . . . . . . 12

a e-mail: alexandre.arbey@ens-lyon.fr

b e-mail: j.auffinger@ipnl.in2p3.fr (corresponding author)

\section{Introduction}

Since Hawking first demonstrated that black holes (BHs) evaporate by emitting a radiation close to the thermal radiation of a black body [1,2], this phenomenon has been extensively studied. One of the main outcome of Hawking radiation (HR) is the possibility that small BHs (i.e. with mass $M_{\mathrm{BH}} \ll M_{\odot}$ ) formed just after the end of the universe expansion, namely primordial BHs (PBHs), may emit or have emitted radiation that could be observable today or that could have left an imprint in cosmology. This leads to a number of constraints on the PBH abundance, depending on their mass $M_{\mathrm{BH}}$, dimensionless spin $a^{*}$, or the extended distribution of both these parameters. For recent reviews on PBH formation and constraints, see Refs. [3,4]. When considering PBHs with mass $M_{\mathrm{BH}} \gtrsim M_{\text {eva }}$ where $M_{\text {eva }} \simeq 5 \times 10^{14} \mathrm{~g}$ is the mass of (Schwarzschild) PBHs evaporating just today if formed at the beginning of the universe, these constraints are given as the fraction of dark matter (DM) $f_{\mathrm{PBH}}$ that the $\mathrm{PBHs}$ represent today. Indeed, light PBHs can represent some fraction of DM as they contribute to cold non baryonic matter in the early universe thermal history [5]. For lighter BHs with mass $M_{\mathrm{BH}} \lesssim M_{\text {eva }}$, these constraints are given as the maximum possible fraction of the universe $\beta$ collapsed into $\mathrm{PBHs}$ at their formation. These BHs cannot represent a significant fraction of DM today since they have evaporated away. However, if evaporation stops at some point, leaving Planck scale remnants, these may contribute to DM [6-11]. To determine the PBH evaporation constraints, one compares the Hawking radiation signals to astrophysical or cosmological observations such as e.g. the extragalactic gamma ray background (EGRB) or the $\Delta N_{\text {eff }}$ limits respectively. PBHs in the mass range just above the evaporation limit, where energy is in the low energy QCD domain $E \sim \mathrm{MeV}$, are severely constrained as a fraction of DM. This represented a difficulty for BlackHawk V1.2, which relied on PYTHIA and HERWIG to compute the hadronization of primary particles, valid for 
$E \gtrsim \mathrm{GeV}$. There was a need for more careful treatment of this energy range, a work initiated by the study of Ref. [12]. Noteworthy, HR is a purely gravitational phenomenon. As such, BHs would radiate any dark sector particle on top of the Standard Model (SM) spectrum, such as supersymmetric states [13], DM or dark radiation (DR) [14-24] - and the graviton [8,19,25-29] (which contributes to DR).

It is thus of utmost importance to be able to predict precisely the HR spectra of SM and beyond SM (BSM) particles. A lot of analytical and numerical results exist in the literature for this purpose, the ones of [30-33] being among the most cited. However, people use most of the time the high-energy limit for the greybody factors given in these papers, resulting in order-of-magnitude constraints. There was thus a need for an automatic program to compute the precise spectra for any BH mass and spin, with a wide choice of parameters that allow for any kind of HR study. This was the context of creation of the public code BlackHawk v1.0 [34]. Since its first release, the code has received some modifications and has reached version 1.2. The previous version of the manual can be found on the arXiv [34] (v2), while the code is publicly available on HEPForge:

\section{https://blackhawk.hepforge.org/}

The code has been recently presented to the TOOLS 2020 conference [35]. BlackHawk is used by many groups from very different domains of astrophysics and cosmology to perform striking studies including, to the knowledge of the authors, evolution of BHs spin [36], EGRB constraints with extended mass distributions and spinning BHs [37] or with higher dimensional Schwarzschild BHs [38], electron and positrons signals from the galaxy with the $511 \mathrm{keV}$ line $[39,40]$, current [41] or prospective [42] X-ray limits, neutrino constraints from Super-Kamiokande [40], JUNO [43] or prospective neutrino detectors $[44,45]$, gamma ray constraints from INTEGRAL [46], COMPTEL with improved low-energy secondary particles treatment [12], prospective AMEGO instrument [12,47], LHASSO [48] or fine modelisation of the Galaxy [49], prediction of signals from Planet 9 within the PBH hypothesis [50], archival galactic center radio observations [51], interstellar medium temperature in dwarf galaxies [52-54] or $21 \mathrm{~cm}$ measurements by EDGES with Schwarzschild [55] or Kerr PBHs [56], Big Bang nucleosynthesis (BBN) [57], heat flow from a small BH captured in the Earth core [58], warm DM from light Schwarzschild [22] and Kerr [23] PBHs, dark radiation from light spinning PBHs [23,24], (extended) dark sector emission [13,59], axion-like particle emission [60], HR from extended BH metrics $[61,62]$. While this release note was being written, the authors became aware of Refs. [63,64] which analyze the production of DM by $\mathrm{PBH}$ evaporation in the early universe; while these do not explicitly use the code BlackHawk, apart from the spin 2 greybody factors, they provide results com- patible with those of BlackHawk with mostly the same spirit in the numerical part.

This paper is a release note of a new version of BlackHawk v2.0 that includes some primordial new features linked to the physics described above: dark sector emission, spin $3 / 2$ greybody factors, BH remnants, BSM BH metrics, low energy hadronization; as well as bug corrections. It then completes the manual published in European Physics Journal C [34] and goes together with the updated version of the code available on the BlackHawk website mentioned above, and an updated version of the manual available on the arXiv [34] (v3). All the installation and run procedures, as well as the complete set of parameters and routines are described in the latter, while we focus here on the new features only. It is organized as follows: in Sect. 2 we describe the physics of the new features added to the code and illustrate them with examples of BlackHawk results, in Sect. 3 we list the new input parameters, in Sect. 4 we mention some improvements on the optimisation of the code and we conclude in Sect. 5. In the following, we use the natural system of units $G=\hbar=c=k_{\mathrm{B}}=1$.

\section{New features}

In this section, we describe the new features added to BlackHawk since the manual was published [34] and we give examples of interesting new results. They have been grouped into distinct categories, even if the changes were made separately: addition of DM, spin $3 / 2$ greybody factors, time dependent features for cosmological studies, greybody factors from new BH metrics, low energy hadronization. Most of these add-ons imply a modification of some parameters and routines, that are listed respectively in Sect. 3 and "Appendix A".

\subsection{Adding a DM particle}

A persistent question of the $\mathrm{BlackHawk}$ users was about the possibility of adding a (BSM) particle to the computed SM spectra. The procedure to implement this modification was described in the BlackHawk manual, but the authors found it very convenient to add this feature to the vanilla version of the code. This addition of a new particle was used in warm DM [22,23] and DR [24] studies where it allowed precision improvement compared to previous analytical approximations.

We need to understand what changes when a particle beyond the SM is Hawking radiated. In fact, as the process is purely gravitational and as the emission of individual particles is independent, any number of additional degrees of freedom can be added without changing the rest of the spectra. The only modification is at the level of the 
$\mathrm{BH}$ lifetime. Indeed, in the integration over the total spectrum to obtain the mass and spin change of the $\mathrm{BH}$, additional dofs provide additional contributions: the mass and spin loss rates are enhanced, thus the $\mathrm{BH}$ evaporates faster. When considering only one additional dof, as in [22-24], the change in the lifetime is negligible, but it could be much higher with numerous additional dof as considered in [13]. In BlackHawk, this change has been taken into account in alternative $f\left(M, a^{*}\right)$ and $g\left(M, a^{*}\right)$ tables computed by an updated (and simplified) version of the script fM.c. Tables fM_add*.txt, fM_add*_nograv.txt, gM_add*.txt and gM_add*_nograv.txt are read instead of the usual fM.txt, fM_nograv. txt, gM. txt and gM_nograv. txt, for the addition of a single massless dof of spin *. The tables $f\left(M, a^{*}\right)$ and $g\left(M, a^{*}\right)$ have further been recomputed with increased precision. The greybody factor tables and fits necessary to recompute the $f, g$ tables are also given in the code source. All these files are available in the folder:

$$
\text { /scripts/greybody_scripts/fM }
$$

If the additional particle considered has a rest mass $\mu$ that has to be taken into account, new tables must be computed using the $\mathrm{fM}$. C script to limit the emission of this particle to the usual condition $E>\mu$. Finally, the primary spectrum output is modified by the addition of one column to the file instantaneous_primary_spectra.txt (program BlackHawk_inst) or by a new file DM_primary_ spectrum.txt (program BlackHawk_tot). The additional dof is assumed to have no interaction with the SM. Hence, there is no modification of the SM secondary spectra. If it were the case, the user would have to modify the routines computing the secondary spectra to implement any new branching ratio (e.g. DM decay into SM particles). We want to stress that within BlackHawk, the graviton is not considered as a BSM particle and is already treated separately since BlackHawk v1.0.

Table 1 below shows the lifetime reduction when one adds a single massless (DM) dof to the SM. As expected, the relative change is small, and depends on the new particle spin, with a decreased impact as the spin increases for a Schwarzschild BH ( $\left.\operatorname{spin} a^{*}=0\right)$. On the contrary, when the $\mathrm{BH}$ is close to Kerr extremal ( $\operatorname{spin} a^{*}=0.9999$ ), higher spin new particles have a greater impact compared to the Schwarzschild case. The spin hierarchy of the impact of adding one dof is not inverted, as one could expect knowing that higher spin particles have a rate of emission much higher than lower spin particles for a close to extremal Kerr $\mathrm{BH}$. The reason is that as the $\mathrm{BH}$ evolves, it loses its spin quite fast and behaves like a Schwarzschild BH for most of its lifetime [36], where the low spin particles have the higher impact.

\subsection{Spin $3 / 2$ particle}

When doing DM and DR studies, it became obvious that particles with spin $3 / 2$ may play a role $[22,23]$. These particles could be motivated by e.g. supersymmetric extensions of the SM; one example is the gravitino, massive spin $3 / 2$ superpartner of the graviton.

The Newman-Penrose form of the Rarita-Schwinger equation of motion for the massless spin $3 / 2$ field in Kerr-Newman geometry, the corresponding radial Teukolsky equation, as well as the short range potential $V_{3 / 2}\left(r\left(r^{*}\right)\right)$ obtained following the Chandrasekhar transform of the Teukolsky equation are described in [65-67]. We must then integrate the equation of motion of the spin $3 / 2$ field from the BH horizon to spatial infinity

$\frac{\mathrm{d}^{2} Z}{\mathrm{~d} r^{* 2}}+\left(E^{2}-V_{3 / 2}\left(r\left(r^{*}\right)\right)\right) Z=0$.

The greybody factor for some partial spin-weighted spherical harmonic $s, l$ is given as the probability that the emitted particle reaches infinity, expressed in terms of the wave function amplitudes as

$\Gamma_{s}^{l}=\left|\frac{Z_{\infty}^{\text {out }}}{Z_{\text {hor. }}^{\text {out }}}\right|^{2}$,

where the superscript "out" refers the the outgoing part of the wave. For the moment, in BlackHawk, we have only implemented the Schwarzschild special case of the spin $3 / 2$ Hawking radiation. The massive spin $3 / 2$ particle can be treated as the other spins, by putting an artificial energy cutoff in the Hawking spectrum at the particle rest mass $E>\mu$.

We have computed the greybody factors thanks to a script similar to the ones used for spins $0,1,2$ and $1 / 2$ particles, in the Schwarzschild case. The Mathematica script for spin $3 / 2$ is spin_1.5.m; it has been added to the BlackHawk folder:

\section{/scripts/greybody_scripts/greybody_ factors}

This script performs the integration of Eq. (1) and tabulates the greybody factors summed over the spherical harmonics decomposition of the wave function. For details about this computation see the complete manual [34]. We advise to use as a "spatial infinity" boundary condition at least $r_{\infty}^{*} / r_{\mathrm{H}}>$ $300 / x$, where $x \equiv E r_{\mathrm{S}}$ is the adimensioned energy, for good numerical convergence. This is particularly important for the low energy greybody factors. The script $\mathrm{fM}$. $\mathrm{C}$ has also been updated as already developed in Sect. 2.1 to include spin 3/2 emission in the $\mathrm{BH}$ lifetime computation. 
Table 1 Table of the relative lifetime reduction of a $M_{\mathrm{BH}}=1 \mathrm{~g} \mathrm{BH}$ with spin $a^{*}=\{0,0.9999\}$, with the addition of a single massless dof to the SM. The greybody factors of a spin $3 / 2$ particle emitted by a Kerr BH have not yet been computed

\begin{tabular}{llllll}
\hline & $s=0(\%)$ & $s=1 / 2(\%)$ & $s=1(\%)$ & $s=3 / 2$ & $s=2(\%)$ \\
\hline$a^{*}=0$ & 1.73 & 0.96 & 0.39 & 0.07 & 0.06 \\
$a^{*}=0.9999$ & 1.43 & 0.93 & 0.51 & $\emptyset$ & 0.70 \\
\hline
\end{tabular}

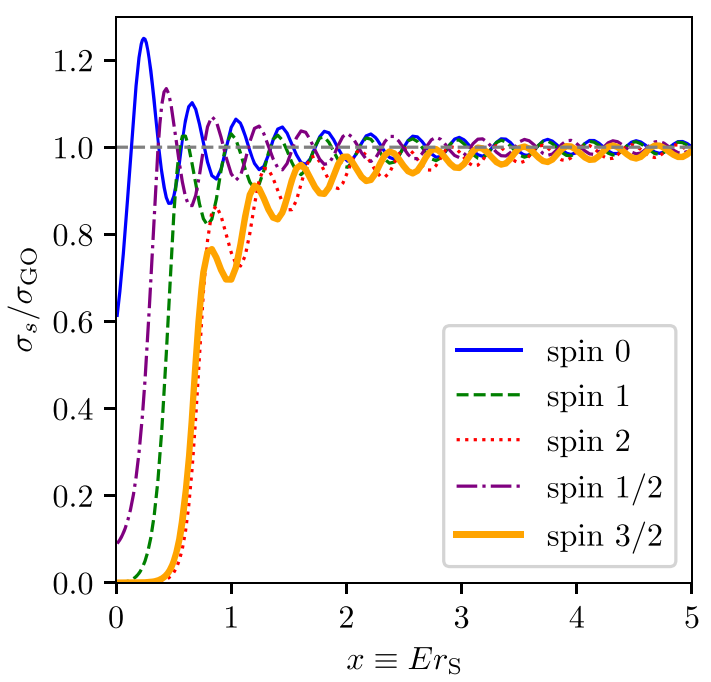

vertical line represents the $\mathrm{BH}$ temperature. These spectra are shape invariant when changing the $\mathrm{BH}$ mass. Right: High energy oscillations of the cross-section, the black horizontal line represents the geometrical optics approximation
In Fig. 1 (left panel) we show the Hawking radiation rate for all massless spins fields with a single dof

$Q_{s}\left(E, M_{\mathrm{BH}}\right) \equiv \frac{\mathrm{d}^{2} N_{s}}{\mathrm{~d} t \mathrm{~d} E}=\frac{\Gamma_{s}}{e^{E / T_{\mathrm{BH}}}-(-1)^{2 s}}$,

where $s$ is the particle spin, $T=1 / 4 \pi r_{\mathrm{S}}$ is the Schwarzschild $\mathrm{BH}$ temperature and

$\Gamma_{s}\left(E, M_{\mathrm{BH}}\right) \equiv \sum_{l=0}^{+\infty}(2 l+1) \Gamma_{s}^{l}\left(E, M_{\mathrm{BH}}\right)$,

where $\Gamma_{s}^{l}$ is the greybody factor for angular momentum $l$, energy $E$ and $\mathrm{BH}$ mass $M_{\mathrm{BH}}$. In the same figure (right panel) we also show the high energy limit of the cross section for all spins

$\sigma_{s} \equiv \frac{\pi \Gamma_{s}}{E^{2}}$,

compared to the geometrical optics (GO) approximation [30]

$\sigma_{\mathrm{GO}} \equiv \frac{27}{4} \pi r_{\mathrm{S}}^{2}$,

where $r_{\mathrm{S}} \equiv 2 M$ is the Schwarzschild horizon radius.

\subsection{Time dependent features, cosmological studies}

Several studies performed with BlackHawk consider Hawking radiation through cosmological eras, e.g. the early universe before BBN [22-24]. It can be relevant to consider not the full time-dependent spectrum of emitted particles, but only the stacked spectrum of those particles at some time after the $\mathrm{BH}$ evaporation. This quantity must be computed by taking the redshift into account. Indeed, particles emitted at the beginning of the $\mathrm{BH}$ evaporation see their energy diluted compared to those emitted at the end of the $\mathrm{BH}$ lifetime. We thus provide into BlackHawk a C script stack. C that performs this integration, with adjustable cosmology parameters such as the matter/radiation domination at the time of evaporation, the time of matter-radiation equality, etc. This script is available in the folder:

$$
\text { /scripts/cosmology_scripts }
$$

and is accompanied by a README. txt file containing instructions. This kind of integration requires that we can extract the time steps of the $\mathrm{BH}$ evaporation precisely. It was not possible with the previous version of BlackHawk as only the absolute time was available in the output files, with 
a exponential precision of 5 digits. At the end of the $\mathrm{BH}$ evaporation, the time steps become so tiny that this precision is not sufficient to distinguish the time steps, making the integration procedure incomplete. Thus, we have added the output file dts.txt to the result set of a BlackHawk run which contains the explicit time steps on top of the absolute time. This file is read by the script stack. c.

The script computes the integrated spectrum $\tilde{Q}_{i}$ of particle $i$ from BH formation $t_{\text {form }}$ to total evaporation $t_{\mathrm{eva}}$

$\tilde{Q}_{i}(E)=\int_{t_{\text {form }}}^{t_{\mathrm{eva}}}(1+z(t)) \frac{\mathrm{d}^{2} N_{i}}{\mathrm{~d} t \mathrm{~d} E}(t,(1+z(t)) E) \mathrm{d} t$.

The redshift between running time $t$ and the evaporation time $t_{\text {eva }}$ is taken into account to shift the energies as well as the BH distribution dilution. This results in the two factors $1+z(t)$ where the redshift as a function of time is given by

$1+z_{\mathrm{RD}}(t)=\left(\frac{t_{\mathrm{eva}}}{t}\right)^{1 / 2}, \quad$ or $1+z_{\mathrm{MD}}(t)=\left(\frac{t_{\mathrm{eva}}}{t}\right)^{2 / 3}$,

for radiation domination (RD) and matter domination (MD) respectively. The case of a mixed or alternate domination of radiation and matter could be straightforwardly implemented in the routine redshift ( ) inside that script.

On top of that, it has become important to treat precisely the boundaries of integral (7). This is why we have implemented in BlackHawk an explicit formula for the value of the BH formation time $t_{\text {form }}$ (see e.g. [3,22])

$t_{\text {form }}=\frac{M_{\mathrm{BH}}}{\gamma}$,

where $\gamma \simeq 0.2$ is the fraction of a Hubble sphere that collapses into a $\mathrm{BH}$ when the density perturbation re-enters the Hubble horizon. This formula is valid for BH formation during a radiation dominated era.

In Fig. 2 we show the (massless) graviton spectrum of a Schwarzschild BH stacked over its lifetime considering RD or MD. The shape can be compared to the instantaneous (massless) spin 2 spectrum of Fig. 1 (left panel).

On a related subject, multiple scenarios of quantum gravity predict that the evaporation of BHs stops at some mass greater than the Planck mass. What is left of the BHs, commonly denoted as $\mathrm{BH}$ relics or $\mathrm{BH}$ remnants, not to mix up with DM relics, does not evaporate and keeps a constant mass. If the $\mathrm{BH}$ was originally spinning or had an electric charge, it is possible that random fluctuations at the end of evaporation attribute a non-zero spin/charge to the remnant [68]. It is also possible that the usual HR paradigm breaks down at some mass scale above the Planck mass, where quantum effects are believed to be important, thus advocating for cautious treatment of the emission below this mass scale. These stable BH remnants, of a mass of the order of magnitude of or superior to the Planck mass, can constitute some fraction

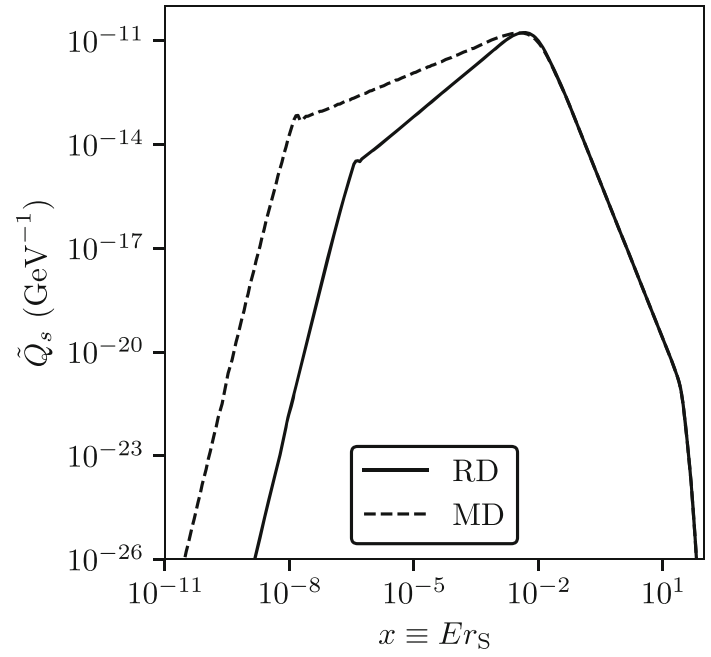

Fig. 2 Stacked graviton spectrum emitted by a $M_{\mathrm{BH}}=10^{-1} \mathrm{~g}$ Schwarzschild $\mathrm{BH}$, in the case of radiation domination (RD, solid black) or matter domination (MD, dashed black)

of DM [6-11] (see however the new discussion started by [69]). As the remnant mass depends on the quantum gravity model, and in particular on the number of extra spatial dimensions [70], we are agnostic about it and leave it as a free parameter of the code. Apart from the particular case of $M_{\text {remnant }} \lesssim M_{\mathrm{BH}}$, the $\mathrm{BH}$ lifetime and stacked spectra should not be perturbed much by the incomplete evaporation.

\subsection{New BH metrics and greybody factors}

Constraints over the PBH fraction of DM are more and more stringent. Would an experiment detect a signal compatible with PBH Hawking radiation, this would constitute a test of the $\mathrm{BH}$ quantum behaviour close to the horizon complementary to the study of $\mathrm{BH}$ quantum normal modes during the ringdown phase after a merger (see e.g. [71]). Then, one needs to know precisely what is the effect of exotic BH metrics on the HR signals. In that context, we have implemented greybody factors computed with different $\mathrm{BH}$ metrics in BlackHawk, namely higher dimensional BHs, charged (Reissner-Nordström) BHs and polymerized BHs. The first were already studied in [72], and more recently Ref. [38] added the higher dimensional greybody factors inside BlackHawk to re-evaluate the extragalactic gamma ray background constraints. Polymerized BHs are one example of a regular $\mathrm{BH}$ solution to the Einstein equations that exhibits a horizon with Hawking radiation. Hence, their HR signals, as well as those of other regular BH solutions, are of particular interest and are the subject of numerous recent studies (see e.g. [73-77]). Some analytical and numerical results about HR from polymerized BHs were presented in [78-82]. The literature on charged BHs is older as they are not astrophysically motivated [68]. We completed those results 


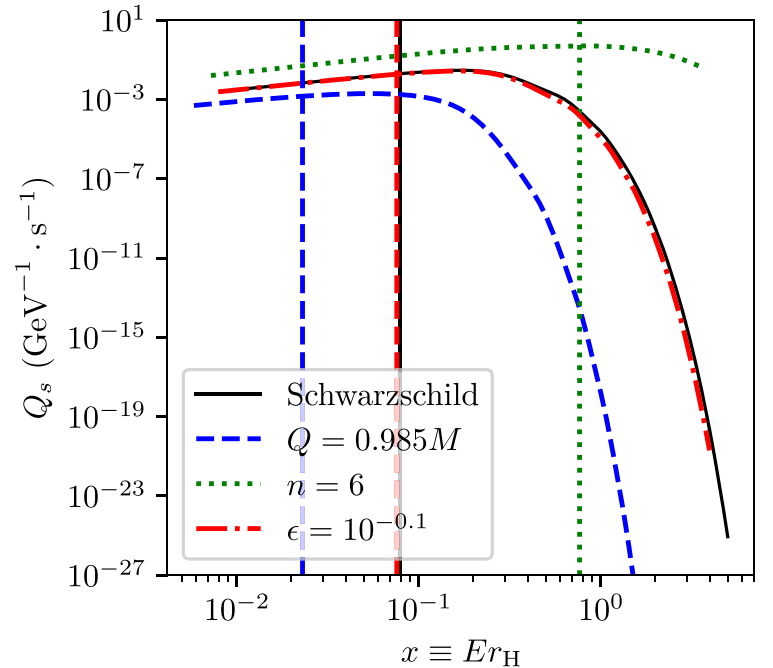

Fig. 3 Comparison of the HR of a massless spin 0 particle from a charged $\mathrm{BH}$ with charge $Q=0.985 \mathrm{M}$ (dashed blue), a higherdimensional $\mathrm{BH}$ with $n=6$ (dotted green) and a polymerized $\mathrm{BH}$ with $\epsilon=10^{-0.1}$ and $a_{0}=0.11$ (dot-dashed red) to a Schwarzschild $\mathrm{BH}$ (solid black) of the same mass $M_{\mathrm{BH}}$. The horizontal axis is labelled

with a full analytical study of the HR by spherically symmetric $\mathrm{BHs}$ described by metrics of the type

$\mathrm{d} s^{2}=-G(r) \mathrm{d} t^{2}+\frac{1}{F(r)} \mathrm{d} r^{2}+H(r) \mathrm{d} \Omega^{2}$,

which are a subset of Petrov type D metrics. In these metrics, the 4-dimensional angular part is $\mathrm{d} \Omega^{2}=\mathrm{d} \theta^{2}+\sin (\theta) \mathrm{d} \varphi^{2}$. We derived the short range potentials $V_{s}$ for the Schrödingerlike wave equation equivalent to the radial Teukolsky equation, for massless fields of spins $0,1,2$ and $1 / 2$ in Ref. [61]. We presented the Hawking radiation spectra in the companion paper [62]. In the literature, there existed a lot of results for $t r$-symmetric metrics of the common type

$F(r)=G(r) \equiv h(r), \quad$ and $H(r)=r^{2}$

Examples include higher dimensional BHs (emission on the brane), charged BHs, (A)dS BHs, etc. The general case where $F(r) \neq G(r)$ received attention only recently when regular $\mathrm{BH}$ solutions of that kind were derived; we computed the greybody factors for polymerized $\mathrm{BHs}$ which are one physically motivated example within the loop quantum gravity (LQG) paradigm. The greybody factors for these metrics are computed in the same manner as the Kerr ones in the previous version of the code. The equations of motion of the massless fields of spins $0,1,2$ and $1 / 2$ (i.e. the NewmanPenrose equations) are separated into a radial and an angular part. The radial equation can then be transformed into a Schrödinger-like wave equation with a short range potential

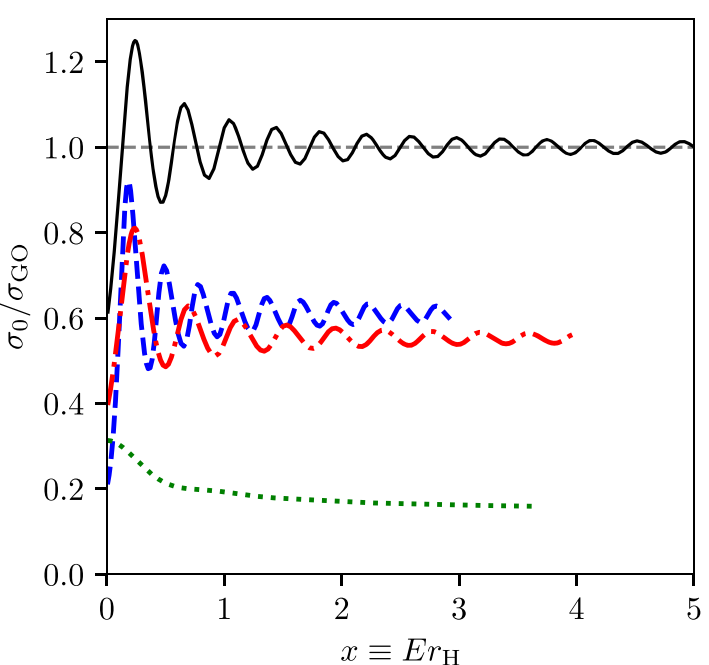

$x \equiv E r_{\mathrm{H}}$ where $r_{\mathrm{H}}$ is the $\mathrm{BH}$ exterior horizon in the corresponding metric. Left: The full Hawking radiation spectra. The vertical lines denote the BH temperatures, with the same color and style correspondence as the curves. Right: The high energy cross section oscillations. The grey horizontal dashed line denotes the geometrical optics approximation

$V_{S}$ depending on the particle spin and the metrics details

$\frac{\mathrm{d}^{2} Z}{\mathrm{~d} r^{* 2}}+\left(E^{2}-V_{s}\left(r^{*}\right)\right) Z=0$.

Solving this equation numerically with planar wave boundary conditions gives access to the reflection and transmission coefficients, the latter being nothing but the greybody factor. Analytical results were obtained in the usual low and high energy limits. For more details about these metrics and their Hawking radiation, see Refs. [61,62].

In Fig. 3, we show the full Hawking spectra for the massless (uncharged) spin 0 scalar in the case of a charged $\mathrm{BH}$, a higher-dimensional $\mathrm{BH}$, and a polymerized $\mathrm{BH}$ (left panel). In the same figure (right panel) we focus on the high energy oscillations of the cross-section.

Inside BlackHawk, these new metrics and their modified greybody factors and $f$ Page factors are available through the parameters.txt file with the new parameter metric. The script $\mathrm{fM}$. $\mathrm{C}$ has been modified to include the possibility of computing the function $f\left(M, \epsilon, a_{0}\right)$ for polymerized BHs, for 11 values of $\epsilon \in[0,0.79]$ and $a_{0}=\{0,0.11\}$, and 11 values of $\epsilon \in[1,100]$ and $a_{0}=0$, where the modified Page factor is given by

$$
\begin{aligned}
f\left(M_{\mathrm{BH}}, \epsilon\right) & \equiv-M_{\mathrm{BH}}^{2} \frac{\mathrm{d} M_{\mathrm{BH}}}{\mathrm{d} t} \\
& =M^{2} \int_{0}^{+\infty} \frac{E}{2 \pi} \sum_{i} \sum_{\mathrm{dof}} \frac{\Gamma_{i}\left(E, M_{\mathrm{BH}}, \epsilon, a_{0}\right)}{e^{E / T_{\mathrm{LQG}}}-(-1)^{s_{i}}} \mathrm{~d} E,
\end{aligned}
$$


where $\Gamma_{i}\left(E, M_{\mathrm{BH}}, \epsilon, a_{0}\right)$ is the modified greybody factor for the polymerized metrics with parameters $\epsilon$ and $a_{0}$. Contrary to the Kerr case, where the BH spin $a^{*}$ evolves during evaporation, here $\epsilon$ and $a_{0}$ are purely constant. We want to stress a particular point: if the polymerized $\mathrm{BH}$ mass gets too small, namely if $r_{+}(M, \epsilon)^{2} \sim a_{0}$, then the validity of the solution is yet to be investigated and we do not guarantee the reliability of the Hawking radiation results. The $f$ tables have been computed with and without the graviton contribution and are stored in:

$$
\text { /src/tables/fM_tables/fM_LQG_*.txt }
$$

On the same model, the greybody factors for the charged BH have been computed for 50 values of $Q \in[0,0.999]$ ( $Q$ is taken as positive, without loss of generality as the greybody factors are computed with neutral particles; the difference with charged particles is altogether of some percent [68]) and for the higher dimensional $\mathrm{BH}$ for up to 6 extra dimensions $n \in\{0,1,2,3,4,5,6\}$ and $M_{*}=1$, with in each case 200 values of $x \equiv 2 E M$ distributed with a new more balanced spread between low and high energies compared to the Kerr case. The Mathematica scripts used to compute them are available in the folders:

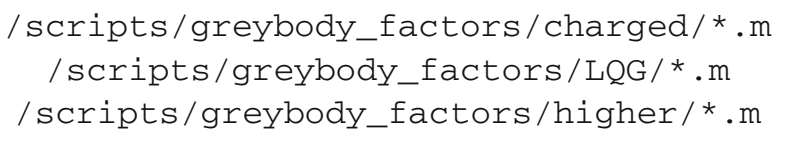

accompanied by Python scripts exploitation.py to produce the fitting tables for the high and low energy limits.

It is of course possible to mix the new metrics with DM contribution to the Hawking spectrum (in that case, the Page factor $f$ must be recomputed for polymerized $\mathrm{BHs}$ ) following the details of Sect. 2.1, at the exception of the spin 3/2 additional particle, for which the greybody factors have not been computed within the new metrics. Using the precise Hawking radiation rates for primordial black holes, Ref. [62] derived the first ever constraints on polymerized primordial BHs as DM.

\subsection{Low energy photon spectrum}

It was obvious during the development of BlackHawk that secondary spectra would cause a difficulty due to the limits in the theoretical and numerical tools at hand to determine the evolution of SM particles after having been Hawking radiated. In the first version of the code, we used the public particle physics codes PYTHIA [83] and HERWIG [84] to convolve the primary spectra with hadronization and decay branching ratios. These codes are designed to match the data of particle accelerators and thus their domain of validity corresponds to the energy range of these accelerators, something like $\sim 5 \mathrm{GeV}$ to $\sim 10 \mathrm{TeV}$. We decided to extend this range to $100 \mathrm{TeV}$, meaning that there is no unknown physics until there (the fundamental interactions are the same, there is no new (interacting) particle in this range). For higher and lower energies, we decided to extrapolate the branching ratios of PYTHIA and HERWIG, when the secondary particles were kinematically allowed. However this should break down at low energy. Indeed, as stated in $[30,31]$, the secondary spectra hypothesis is that primary SM particles are emitted at all energies if kinematically allowed $(E>\mu$ where $\mu$ is the particle rest mass), then these fundamental particles are hadronized or decayed. For example, a down quark with a mass of $\simeq 4.7 \mathrm{MeV}$ can be emitted at an energy of $E>4.7 \mathrm{MeV}$. But as we know, quarks can not exist outside of bound states, and the lightest bound state is the neutral pion with $\mu_{\pi^{0}} \simeq 135 \mathrm{MeV}$. The questions is now: are quarks of type $q$ Hawking emitted between $E=\mu_{q}$ and $E=\mu_{\pi^{0}}$ ? A question which has been reformulated elsewhere as: what are the fundamental particles at some low energy $E$ ? Indeed, we can argue that below some energy scale (typically the QCD scale), the fundamental degrees of freedom of the theory are the pions and not the individual quarks. Below the pion rest masses, there is no viable QCD degree of freedom, even if light quarks and gluons are kinematically allowed. That is the scheme chosen by [12] when deriving the secondary photon spectrum at low energy (below the QCD scale).

We thus modified BlackHawk such that it emits pions instead of single quarks if the user chooses so, with the new hadronization choice in the hadronization_choice parameter. Then, we take back the development of [12] on the behaviour of the particles at low energy and use the public Python code Hazma [85] to evolve the primary particles. We restrict ourselves to the secondary photon and electron spectra (Hazma does not provide neutrino spectra), which is an improvement over [12] which only considered secondary photons. Depending on the available energy, electrons, muons and pions can contribute to the secondary spectra, in addition to the primary photons and electrons. The contributions are:

- final state radiation (FSR): pairs of created opposite charge particles radiate photons, this concerns electrons, muons and charged pions;

- decays: single unstable particles decay into photons or electrons, this concerns muons, neutral and charged pions.

We follow [12] to compute the FSR photons thanks to the electroweak splitting functions [86] directly inside BlackHawk from the primary electron, muon and charged pions spectra. We also follow [12] and use the public code Hazma [85] to compute the low-energy pions and muon decay rates, which we have tabulated in:

$$
\text { /src/tables/hazma_tables/photon.txt }
$$


which covers center of mass energy of $1 \mathrm{keV}$ to $5 \mathrm{GeV}$. We improve on [12] by including the secondary low energy electron and positron spectra, from the decay rates of muons and charged pions tabulated in:

\section{/src/tables/hazma_tables/electron.txt}

The Python script we have used to compute these tables is available at:

$$
\text { /scripts/hazma_scripts/hazma_tables.py }
$$

Then, we add up all the contributions to the primary photon and electron spectra to obtain the total secondary spectra.

There are several drawbacks to this methodology. First, when FSR occurs, there is a loss of center of mass energy into photons, which should alter the charged particles spectra before decays, even if at second order in the fine structure constant. That difficulty arises because the Hazma code convolves initial spectra with analytical transfer functions and does not perform a MCMC statistical analysis of competing processes. Second, and this is the main difficulty with the QCD phenomenology, it is not clear what is precisely the QCD scale $\Lambda_{\mathrm{QCD}}$ (between $\mu_{\pi^{0}}$ and $300 \mathrm{MeV}$ ) that separates direct pion emission from single quarks and gluons emission that hadronize into jets. This is the reason why we do not fix a $\Lambda_{\mathrm{QCD}}$ at some value, but rather provide both hadronization possibilities. The spectra should interpolate between the two limits when the center of mass energy explores the QCD range. As we can not decide (outside of a chosen model) what are the number of degrees of freedom and their nature in the QCD energy range, this hadronization method is limited to the program BlackHawk_inst, because we need this information to compute the Page evolution factors inside BlackHawk_tot. The PBH masses at stake are $M_{\mathrm{PBH}} \gtrsim 10^{14} \mathrm{~g}$, thus we can consider that these PBHs are living indefinitely at our time scale - the age of the universe - and that BlackHawk_inst is enough for $\mathrm{PBH}$ studies linked to photon or electron emission in this scheme.

We show in Fig. 4 (respectively Fig. 5) the total photon (respectively electron) spectrum computed with the old extrapolated version of BlackHawk, compared to the new spectra computed with the method of [12] for a $\mathrm{BH}$ of mass $5.3 \times 10^{14} \mathrm{~g}$.

We point out that this new feature is dedicated to the low energy photon and electron emission, and does not for now provide low energy spectra for neutrinos, which however are only related to sub-dominant (but complementary) constraints in the considered PBH mass range [40,43-45]. We stress that the new spectra may alter the constraints of previous gamma ray and electron-positron studies from Hawking evaporation in the narrow mass range $5 \times 10^{14} \mathrm{~g} \lesssim M_{\mathrm{PBH}} \lesssim$ $10^{16} \mathrm{~g}$, where PBHs can only represent a negligible fraction of DM anyway $[3,4]$.

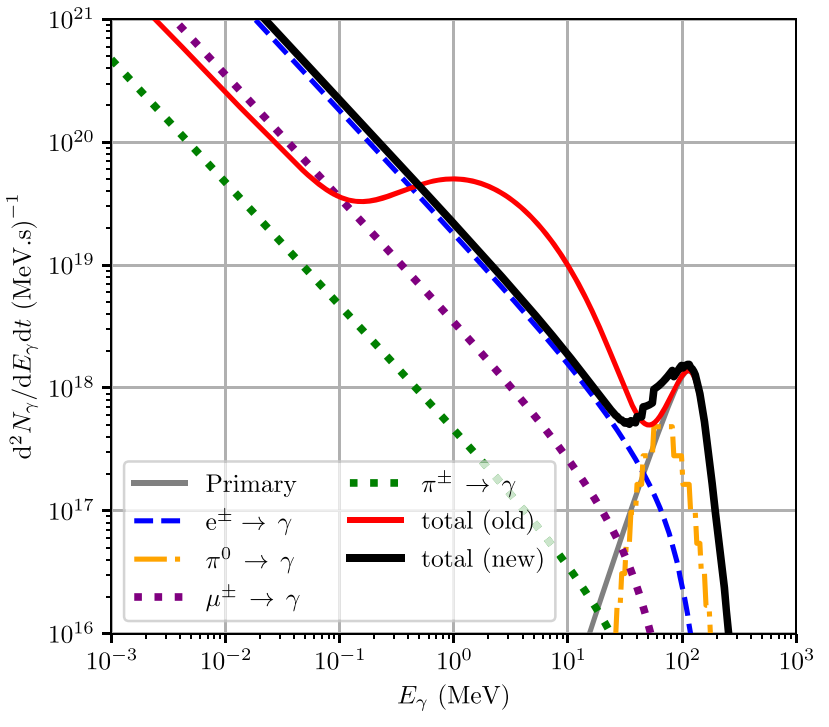

Fig. 4 Comparison between the old PYTHIA extrapolated (solid red) and new Hazma computed (solid black) BlackHawk instantaneous total photon spectrum for a $M_{\mathrm{BH}}=5.3 \times 10^{14} \mathrm{~g}$. From left to right: primary photon spectrum (grey solid); neutral pion decay (orange dotdashed); electron FSR (blue dashed); muon decay+FSR (purple dotted); charged pion decay+FSR (green dotted). To be compared with Fig. 2 upper left panel of [12]

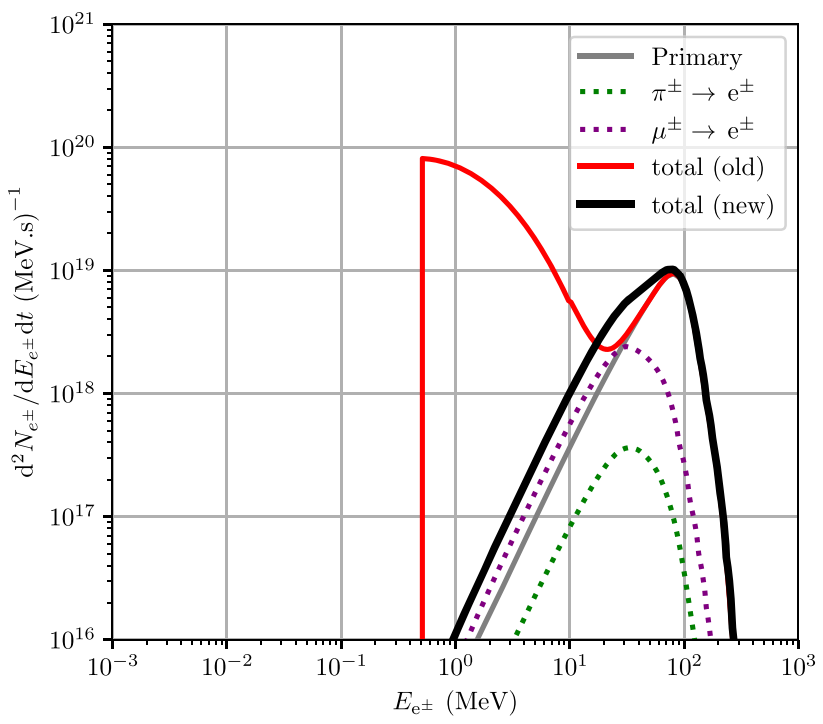

Fig. 5 Comparison between the old PYTHIA extrapolated (solid red) and new Hazma computed (solid black) BlackHawk instantaneous total electron spectrum for a $M_{\mathrm{BH}}=5.3 \times 10^{14} \mathrm{~g}$. From left to right: primary electron spectrum (grey solid); muon decay (purple dotted); charged pion decay (green dotted)

\section{Parameters}

In this section we describe the new parameters.txt file resulting from the modifications and add-ons described in Sect. 2, as well as a simplification of the presentation of the file by a removal of "hard-coded" parameters such 
as the dimensions of the numerical tables. These parameters are read by new routines in new infos. txt files. On another hand, as we have added new metrics, some parameters changed their name from *_a (Kerr case) to *_param in order to be generalized. As a consequence, the extended distributions for the $\mathrm{BH}$ spin $a^{*}$ have been adapted to the $\mathrm{BH}$ charge $Q$, while for polymerized and higher dimensional $\mathrm{BHs}$ there is only one value of the secondary parameter allowed for each run: (a_number $\rightarrow$ ) param_number $=1$. We focus here only in the new or modified parameters which are the interesting entries for BlackHawk users. For a complete description of the new and modified routines see "Appendix A" in this paper or the complete updated BlackHawk v2 . 0 manual [34]. Some bug corrections are listed in "Appendix B". The parameters file/structure have several interesting new entries:

- the new parameter metric switches between the Kerr metric (0), the polymerized metric (1), the ReissnerNordström metric (2) and the higher-dimensional metric (3);

- for the new BH metrics, we have added the parameters Qmin and Qmax (dimensionless - positive - BH charge between 0 and 1), epsilon_LQG and a0_LQG (polymerized metric, $\epsilon$ being the polymerization parameters between 0 and 1 and $a_{0}$ the minimal dimensionless area), and finally $\mathrm{n}$ and $\mathrm{M} \_$star (higher dimensional metric, $n$ is the number of extra dimensions and $M_{*}$ the rescaled Planck mass);

- tmin_manual: as described in Sect. 2.3, there is now a possibility to choose between a manual $\mathrm{BH}$ formation time $t_{\text {form }}\left(\right.$ tmin_manual $\left.=1, \operatorname{tmin}=t_{\text {form }}\right)$ and the fidutial $t_{\text {form }}$ given by Eq. (9) (tmin_manual = 0 ), the latter being only relevant for monochromatic $\mathrm{BH}$ distributions and radiation domination at formation;

- nb_final_times has been removed as it is now fixed by the integration procedure;

- nb_gamma_spins describes how many particle spins are available for some BH metric, and nb_gamma_fits encodes the number of fitting parameters used to extend the greybody factor tables to low and high energy;

- add_DM, m_DM, spin_DM and dof_DM are new parameters linked to the DM emission described in Sect. 2.1. To add DM emission one has to set add_DM $=1$ (otherwise let it to 0 ), then one can choose the DM mass $m_{\mathrm{DM}}$ in $\mathrm{GeV}$, the DM spin $s_{\mathrm{DM}} \in\{0,1,2,0.5,1.5\}$ and the DM number of dof (be carefull that only 1 dof has been taken into account when computing the new $f, g$ tables with DM emission);

- BH_remnant switches between the usual total evaporation at Planck mass (BH_remnant $=0$ ) and an interrupted evaporation at M_remnant $\geq M_{\mathrm{P}}\left(\mathrm{BH} \_r\right.$ remnant $=1$ );
- the parameter hadronization_choice can now take the value 3 , corresponding to the hadronization procedure described in Sect. 2.5. This choice forces the DM parameters to be used to compute the pion spectrum, thus the low energy hadronization procedure is at present incompatible with DM emission by BHs;

- the Page factors tables parameters (Mmin_fM, Mmax_fM, nb_fM_masses and nb_fM_a $\rightarrow$ nb_fM_param), the greybody factor tables parameters (particle_number, nb_gamma_a $\rightarrow$ nb_gamma _param, nb_gamma_x, a_min $\rightarrow$ param_min and a_max $\rightarrow$ param_max) and the hadronization parameters (Emin_hadro, Emax_hadro, nb_init_en, nb_fin_en, nb_init_part and nb_fin_part) do not appear anymore in the parameters. txt file as they are $a$ priori fixed, they are now read in info files stored in respectively:

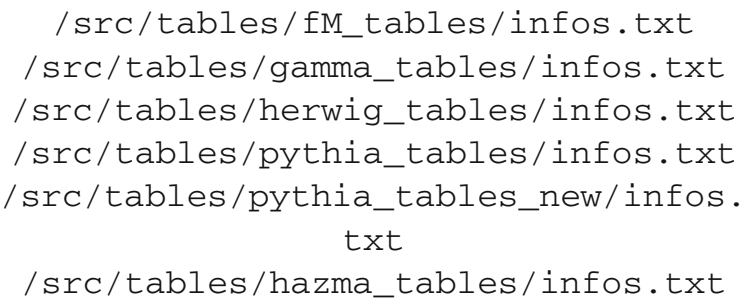

and we recommend that they be not modified unless the tables are recomputed;

- the following parameters changed their name: BHnumber $\rightarrow$ BH_number, Enumber $\rightarrow$ E_number, anumber $\rightarrow$ param_number.

\section{Optimisation}

In this section we briefly present a simple optimisation procedure that has been implemented inside BlackHawk in order to fasten the computations and diminish the disc memory occupied by the output files when some specific studies do not necessitate the full Hawking radiation spectra. Following the addition of the write_* parameters which decide whether some particle spectrum is written down to the output, we added the tables compute_primary [] and compute_secondary[] which allow the code to skip some particle spectrum computations. We must warn the user that if some primary particle "computation" parameter is set to 0 , then this particle will not participate in the secondary spectra as well. However, for studies focused on a single particle of some type, e.g. the graviton, DM or some secondary particle, then the other secondary particles can be "turned off". The BH time evolution will not be affected as it is determined by the numerical tables of the Page factors $f, g$. 


\section{Conclusion}

We have described the new features available in the public code BlackHawk v2.0, together with interesting illustrations: the addition of dark matter (and dark radiation) emission, the massless spin $3 / 2$ field greybody factors for the Schwarzschild metric, the possibility to keep a black hole remnant at the end of evaporation, the greybody factors associated with spherically symmetric and static black hole solutions more general than the Schwarzschild case and the careful computation of the low energy photon and electron spectra. We hope that this will open a new era of Hawking radiation studies with already promising results. An updated version of the complete manual is available on the arXiv [34] (v3) and the BlackHawk v2 . 0 code is available on HEPForge at https://blackhawk.hepforge.org/.

Data Availability Statement This manuscript has no associated data or the data will not be deposited. [Authors' comment: There is no data that goes with the manuscript; the figures are self-consistent and there is no additional data.]

Open Access This article is licensed under a Creative Commons Attribution 4.0 International License, which permits use, sharing, adaptation, distribution and reproduction in any medium or format, as long as you give appropriate credit to the original author(s) and the source, provide a link to the Creative Commons licence, and indicate if changes were made. The images or other third party material in this article are included in the article's Creative Commons licence, unless indicated otherwise in a credit line to the material. If material is not included in the article's Creative Commons licence and your intended use is not permitted by statutory regulation or exceeds the permitted use, you will need to obtain permission directly from the copyright holder. To view a copy of this licence, visit http://creativecomm ons.org/licenses/by/4.0/.

Funded by SCOAP ${ }^{3}$.

\section{Appendix A: Routines}

In this section we list the new and modified routines of the two programs BlackHawk_inst and BlackHawk_tot, resulting from the new features described in Sect. 2, as well as the modified parameters listed in Sect. 3. We do not list the modifications to the header file include. $h$ and compilation file Makefile as they are transparent regarding the content of Sect. 3 and of the present section.

\section{Modified routines}

The modifications of existing routines are:

- int read_params (struct param *parameters, char name[], int session) has been modified to read the new parameters described in Sect. 3 and received bug corrections when parameters were in conflict, as well as a new error display;

- int memory_estimation(struct param *parameters, int session) has been updated to take into account the new BlackHawk features (e.g. the addition of DM to the number of particles simulated);

- void read_gamma_tables (double ***gammas, double *gamma_a, double * gamma_x, struct param

* parameters )

void read_asymp_fits

(double ***fits, struct param *

parameters) now include the spin $3 / 2$ greybody factor tables;

- void instantaneous_primary_spectrum (double **instantaneous_primary_ spectra, double **BH_masses, double ** BH_spins, double **spec_table, double *energies, double ***gammas, double * gamma_a, double *gamma_x, double *** fits, double *dof, double spins, *double *masses_primary, int **Counters_a, int $* * *$ counters_x, int ${ }^{*}$ compute, struct param *parameters) now takes as arguments the tables counters_x[], counters_a[] and compute[] (for the latter see Sect. 4) to avoid re-definition of those tables at each time step in the program BlackHawk_tot;

- void write_instantaneous_primary _spectraldouble **instantaneous_ primary_spectra, double *energies, struct param *parameters), void write_instantaneous_secondary_ spectra (double**instantaneous_ integrated_hadronized_spectra, double *hadronized_energies, struct param * parameters) and void write_lines(char **file _names_primary, char ** file_names_secondary, double **partial _primary_spectra, double **partial _integrated_hadronized_spectra, int *write_primary, int *write_secondary, double time, struct param *parameters) write down the spectrum of DM if add_DM $=1$;

- void total_spectra(double ***partial_ hadronized_spectra, double **partial_ primary_spectra, double **partial _integrated_hadronized_spectra, double ****tables, double *initial_energies, double *final_energies, double **spec_ table, double *times, double $* * *$ life 
masses, double **BH_masses, double $\star * \star$ life_spins, double $* * \mathrm{BH}_{\mathrm{B}}$ spins, double *energies, double *masses_ primary, double *spins, double *dof, double *masses_secondary, double ***gammas, double *gamma_a, double *gamma_x, double

***fits, struct param *parameters) now takes into account the computation of the DM spectrum if add_DM = 1, it takes the tablesmasses_primary [ ] spins [ ] and dof [] as arguments to homogenize the structure of the code and defines the tables compute _primary [] and compute_secondary [] (see Sect. 4);

- void life_evolution(double ***life -masses, double $* * \star l i f e \_s p i n s, ~ d o u b l e$ *Iife_times, double *dts, double *init _masses, double *init_spins, int

**rank, double **fM_table, double **gM_table, double *fM_masses, double *fM_a, struct param *parameters) fills a table dts [] containing the time steps of integration that are useful for precise time dependent studies (see Sect. 2.3);

- the routines void read_hadronization_infos (struct param *parameters), void read_hadronization_tables (double ****tables, double *initial_energies, double *final_energies, struct param *parameters), double contribution -instantaneous(int $j$, int counter, int $k$, double **instantaneous_primary _spectra, double ****tables, double *initial_energies, double

*final_energies, int particle_type, struct param *parameters) and void hadronize_instantaneous (double ***instantaneous_hadronized_spectra, double ****tables, double *initial_ energies, double

*final_energies, double

* *instantaneous_primary_spectra, double *energies, double *masses_secondary, int *compute, struct param

*parameters) have been modified to read and use the new hadronization tables computed with Hazma (see Sect. 2.5), while void convert_hadronization _tables (double ****tables, double *initial_energies, double *final_ energies, struct param * parameters) has been extended and used to generate the new header file:

$$
\text { /src/hadro_hazma.h }
$$

compiled within the BlackHawk programs if HARDTABLES is defined.

We have modified the evolution routines in order to account for the new BH metrics, associated with so-called $\mathrm{BH}$ "secondary parameters" (the BH charge, the value of $\epsilon$ for polymerized BHs or the number of extra dimensions). In fact, all these parameters behave in the same manner as the spin. Some can even be treated as constants, like the number of extra dimensions.

Most routines also take into account the fact that the number of primary particles is now given by:

$$
\text { particle_number + grav + add_DM }
$$

\section{New routines}

The new routines are:

- int read_fM_infos (struct param * parameters) reads the $f, g$ tables info in the new file:

\section{/src/tables/fM_tables/infos.txt}

and fills the parameters Mmin_fM, Mmax_fM, nb_fM _masses and nb_fM_param;

- int read_gamma_infos (struct param * parameters ) reads the greybody factors tables info in the new files:

\section{/src/tables/gamma_tables/infos.txt}

and fills the parameters particle_number, nb_ gamma_param, nb_gamma_x, param_min and param_max;

- int read_hadronization_infos (struct param * parameters) reads the hadronization tables info in one of the new files:

/src/tables/herwig_tables/infos.txt

/src/tables/pythia_tables/infos.txt /src/tables/pythia_tables_new/infos.txt

/src/tables/hazma_tables/infos.txt

depending on the hadronization_choice parameter;

- the routine void write_life_evolutions (double $* * *$ life_masses, double ***life _spins, double *life_times, double * dts, struct param * parameters) writes down the new output file dts. txt (see Sect. 2.3); 
- new temperature functions double temp_Kerr (double M, double a), double temp_LQG (double M, double epsilon, double a0), double temp_charged(double M, double Q) and double temp_higher(double M, double $\mathrm{n}$, double M_star) have been written to compute the Hawking temperature for the new $\mathrm{BH}$ metrics, as well as some secondary necessary functions double rplus_Kerr(double M, double a), double P_LQG

(double epsilon), double m_LQG (double M, double epsilon), double rplus_charged (double M, double Q), double rminus _charged(double $\mathrm{M}$, double Q) and $r \mathrm{H}$ _higher(double M, double $\mathrm{n}$, double M star);

- void add_FSR_instantaneous (double **instantaneous_primary_spectra, double **instantaneous_integrated_hadronized_ spectra, double *energies, double *final_energies, double *masses_primary, struct param *parameters) computes the FSR of electrons, muons and charged pions as described in Sect. 2.5; we have been careful about the number of degrees of freedom, as for example the "electron" spectrum in BlackHawk in fact takes into account the $\mathrm{e}^{ \pm}$ multiplicity, we have to divide the formulas of [85] by 2 because they also account for it a second time;

- new void free_* ( ) functions have been defined to free arrays of different formats;

- double exp_adapt (double $\mathrm{x}$ ) computes the quantity $\exp (x)-1$ in the case of small $x$, using the Taylor development of the exponential function up to 5 th order.

\section{Appendix B: Corrections}

In this section, we briefly mention the few typo corrections that were spotted in the manual [34] when developing this new version of the code:

- There was a double mistake in the definition of $K$ below Eqs. (2.19) and (2.22). It should be $K \equiv r^{2} E$ and $K \equiv$ $\left(r^{2}+a^{2}\right) E+a m$ respectively, as shows dimensional analysis.

- The results presented in "Appendix D" are computed for $M_{\mathrm{BH}}=10^{10} \mathrm{~g}$, as shows e.g. the density in "Appendix D.2", whereas the file parameters.txt mentions $M_{\mathrm{BH}}=10^{15} \mathrm{~g}$ in "Appendix D.1".

- Between Eqs. (2.13) and (2.14), it is said that the sum over the angular momentum modes $m=-l, \ldots, l$, when all of them give the same contribution to the greybody factor, results in a factor $l(l+1)$. It is of course a factor $2 l+1$.

- As there is an ambiguity on the precise definition of the BH mass distribution denoted as "log-normal", we have decided to add both a "log-normal" distribution for the mass and for the number density of BHs (spectrum_choice = 1 and spectrum_choice $=11$ respectively).

\section{References}

1. S.W. Hawking, Black hole explosions, Nature 248, 30 (1974) (NoStop)

2. S.W. Hawking, Particle creation by black holes, Commun. Math. Phys. 43, 199 (1975). [Erratum: Commun. Math. Phys. 46, 206 (1976)] (NoStop)

3. B. Carr, K. Kohri, Y. Sendouda, J. Yokoyama, Constraints on primordial black holes (2020). arXiv:2002.12778 [astro-ph.CO] (NoStop)

4. A.M. Green, B.J. Kavanagh, Primordial Black Holes as a dark matter candidate. J. Phys. G 48, 4 (2021). arXiv:2007.10722 [astroph.CO] (NoStop)

5. B. Carr, F. Kuhnel, Primordial black holes as dark matter: recent developments. Ann. Rev. Nucl. Part. Sci. 70, 355 (2020). arXiv:2006.02838 [astro-ph.CO] (NoStop)

6. J.H. MacGibbon, Can Planck-mass relics of evaporating black holes close the universe? Nature 329, 308 (1987) (NoStop)

7. J.D. Barrow, E.J. Copeland, A.R. Liddle, The Cosmology of black hole relics. Phys. Rev. D 46, 645 (1992) (NoStop)

8. A.D. Dolgov, P.D. Naselsky, I.D. Novikov, Gravitational waves, baryogenesis, and dark matter from primordial black holes (2000). arXiv:astro-ph/0009407 (NoStop)

9. P. Chen, R.J. Adler, Black hole remnants and dark matter. Nucl. Phys. B Proc. Suppl. 124, 103 (2003). arXiv:gr-qc/0205106 (NoStop)

10. A. Barrau, D. Blais, G. Boudoul, D. Polarski, Peculiar relics from primordial black holes in the inflationary paradigm. Ann. Phys. 13, 115 (2004). arXiv:astro-ph/0303330 (NoStop)

11. B.V. Lehmann, C. Johnson, S. Profumo, T. Schwemberger, Direct detection of primordial black hole relics as dark matter. JCAP 10, 046 (2019). arXiv:1906.06348 [hep-ph] (NoStop)

12. A. Coogan, L. Morrison, S. Profumo, Direct detection of Hawking radiation from asteroid-mass primordial black holes. Phys. Rev. Lett. 126, 171101 (2021). arXiv:2010.04797 [astro-ph.CO] (NoStop)

13. M.J. Baker and A. Thamm, Probing the particle spectrum of nature with evaporating black holes (2021). arXiv:2105.10506 [hep-ph] (NoStop)

14. T. Fujita, M. Kawasaki, K. Harigaya, R. Matsuda, Baryon asymmetry, dark matter, and density perturbation from primordial black holes. Phys. Rev. D 89, 103501 (2014). arXiv:1401.1909 [astroph.CO] (NoStop)

15. O. Lennon, J. March-Russell, R. Petrossian-Byrne, H. Tillim, Black hole genesis of dark matter. JCAP 04, 009. arXiv:1712.07664 [hep$\mathrm{ph}$ ( NoStop)

16. D. Hooper, G. Krnjaic, S.D. McDermott, Dark radiation and superheavy dark matter from black hole domination. JHEP 08, 001 (2019). arXiv:1905.01301 [hep-ph] (NoStop)

17. I. Masina, Dark matter and dark radiation from evaporating primordial black holes. Eur. Phys. J. Plus 135, 552 (2020). arXiv:2004.04740 [hep-ph] (NoStop) 
18. I. Baldes, Q. Decant, D.C. Hooper, L. Lopez-Honorez, Non-cold dark matter from primordial black hole evaporation. JCAP 08, 045 (2020). arXiv:2004.14773 [astro-ph.CO] (NoStop)

19. D. Hooper, G. Krnjaic, J. March-Russell, S.D. McDermott, R. Petrossian-Byrne, Hot gravitons and gravitational waves from Kerr Black Holes in the early universe (2020). arXiv:2004.00618 [astroph.CO] (NoStop)

20. C. Keith, D. Hooper, N. Blinov, S.D. McDermott, Constraints on primordial black holes from Big Bang Nucleosynthesis revisited. Phys. Rev. D 102, 103512 (2020). arXiv:2006.03608 [astro-ph.CO] (NoStop)

21. P. Gondolo, P. Sandick, B. Shams EsHaghi, Effects of primordial black holes on dark matter models. Phys. Rev. D 102, 095018 (2020). arXiv:2009.02424 [hep-ph] (NoStop)

22. J. Auffinger, I. Masina, G. Orlando, Bounds on warm dark matter from Schwarzschild primordial black holes. Eur. Phys. J. Plus 136, 261 (2021). arXiv:2012.09867 [hep-ph] (NoStop)

23. I. Masina, Dark matter and dark radiation from evaporating Kerr primordial black holes (2021). arXiv:2103.13825 [gr-qc] (NoStop)

24. A. Arbey, J. Auffinger, P. Sandick, B. Shams EsHaghi, K. Sinha, Precision calculation of dark radiation from spinning primordial black holes and early matter-dominated eras. Phys. Rev. D 103, 123549 (2021). arXiv:2104.04051 [astro-ph.CO] (NoStop)

25. G.S. Bisnovatyi-Kogan, V.N. Rudenko, Very high frequency gravitational wave background in the universe. Class. Quantum Gravity 21, 3347 (2004). arXiv:gr-qc/0406089 (NoStop)

26. R. Anantua, R. Easther, J.T. Giblin, GUT-Scale primordial black holes: consequences and constraints. Phys. Rev. Lett. 103, 111303 (2009). arXiv:0812.0825 [astro-ph] (NoStop)

27. A.D. Dolgov, D. Ejlli, Relic gravitational waves from light primordial black holes. Phys. Rev. D 84, 024028 (2011). arXiv:1105.2303 [astro-ph.CO] (NoStop)

28. R. Dong, W.H. Kinney, D. Stojkovic, Gravitational wave production by Hawking radiation from rotating primordial black holes. JCAP 10, 034 (2015). arXiv:1511.05642 [astro-ph.CO] (NoStop)

29. K. Inomata, M. Kawasaki, K. Mukaida, T. Terada, T.T. Yanagida, Gravitational wave production right after a Primordial black hole evaporation. Phys. Rev. D 101, 123533 (2020). arXiv:2003.10455 [astro-ph.CO] (NoStop)

30. J.H. MacGibbon, B.R. Webber, Quark and gluon jet emission from primordial black holes: the instantaneous spectra. Phys. Rev. D 41, 3052 (1990) (NoStop)

31. J.H. MacGibbon, Quark and gluon jet emission from primordial black holes. 2. The Lifetime emission. Phys. Rev. D 44, 376 (1991) (NoStop)

32. J.H. MacGibbon, B.J. Carr, Cosmic rays from primordial black holes. Astrophys. J. 371, 447 (1991) (NoStop)

33. F. Halzen, E. Zas, J.H. MacGibbon, T.C. Weekes, Gamma-rays and energetic particles from primordial black holes. Nature 353, 807 (1991) (NoStop)

34. A. Arbey, J. Auffinger, BlackHawk: A public code for calculating the Hawking evaporation spectra of any black hole distribution. Eur. Phys. J. C 79, 693 (2019). arXiv:1905.04268 [gr-qc] (NoStop)

35. J. Auffinger, A. Arbey, BlackHawk: a tool for computing Black Hole evaporation. In booktitle Tools for High Energy Physics and Cosmology (2020). arXiv:2012.12902 [gr-qc] (NoStop)

36. A. Arbey, J. Auffinger, J. Silk, Evolution of primordial black hole spin due to Hawking radiation. Mon. Not. R. Astron. Soc. 494, 1257 (2020). arXiv:1906.04196 [astro-ph.CO] (NoStop)

37. A. Arbey, J. Auffinger, J. Silk, Constraining primordial black hole masses with the isotropic gamma ray background. Phys. Rev. D 101, 023010 (2020). arXiv:1906.04750 [astro-ph.CO] (NoStop)

38. G. Johnson, primordial black hole constraints with large extra dimensions. JCAP 09, 046 (2020). arXiv:2005.07467 [astroph.CO] (NoStop)
39. R. Laha, Primordial black holes as a dark matter candidate are severely constrained by the Galactic Center $511 \mathrm{keV} \gamma$-Ray Line. Phys. Rev. Lett. 123, 251101 (2019). arXiv:1906.09994 [astroph.HE] (NoStop)

40. B. Dasgupta, R. Laha, A. Ray, Neutrino and positron constraints on spinning primordial black hole dark matter. Phys. Rev. Lett. 125, 101101 (2020). arXiv:1912.01014 [hep-ph] (NoStop)

41. C.M. Lee, M.H. Chan, The evaporating primordial black hole fraction in cool-core galaxy clusters (2021). arXiv:2103.12354 [astroph.HE] (NoStop)

42. G. Ballesteros, J. Coronado-Blázquez, D. Gaggero, X-ray and gamma-ray limits on the primordial black hole abundance from Hawking radiation. Phys. Lett. B 808, 135624 (2020). arXiv:1906.10113 [astro-ph.CO] (NoStop)

43. S. Wang, D.-M. Xia, X. Zhang, S. Zhou, Z. Chang, Constraining primordial black holes as dark matter at JUNO. Phys. Rev. D 103, 043010 (2021). arXiv:2010.16053 [hep-ph] (NoStop)

44. R. Calabrese, D.F.G. Fiorillo, G. Miele, S. Morisi, A. Palazzo, Primordial black hole dark matter evaporating on the Neutrino Floor (2021). arXiv:2106.02492 [hep-ph] (NoStop)

45. V. DeRomeri, P. Martínez-Miravé, M. Tórtola, Signatures of primordial black hole dark matter at DUNE and THEIA (2021). arXiv:2106.05013 [hep-ph] (NoStop)

46. R. Laha, J.B. Muñoz, T.R. Slatyer, INTEGRAL constraints on primordial black holes and particle dark matter. Phys. Rev. D 101, 123514 (2020). arXiv:2004.00627 [astro-ph.CO] (NoStop)

47. A. Ray, R. Laha, J.B. Muñoz, R. Caputo, Closing the gap: Near future $\mathrm{MeV}$ telescopes can discover asteroid-mass primordial black hole dark matter (2021). arXiv:2102.06714 [astro-ph.CO] (NoStop)

48. Y.-F. Cai, C. Chen, Q. Ding, Y. Wang, Ultrahigh-energy Gamma Rays and gravitational waves from primordial exotic stellar bubbles (2021). arXiv:2105.11481 [astro-ph.CO] (NoStop)

49. J. Iguaz, P.D. Serpico, T. Siegert, Isotropic X-ray bound on primordial black hole dark matter (2021). arXiv:2104.03145 [astroph.CO] (NoStop)

50. A. Arbey, J. Auffinger, Detecting Planet 9 via Hawking radiation (2020). arXiv:2006.02944 [gr-qc] (NoStop)

51. M.H. Chan, C.M. Lee, Constraining primordial black hole fraction at the galactic centre using radio observational data. Mon. Not. R. Astron. Soc. 497, 1212 (2020). arXiv:2007.05677 [astro-ph.HE] (NoStop)

52. H. Kim, A constraint on light primordial black holes from the interstellar medium temperature (2020). arXiv:2007.07739 [hep$\mathrm{ph}$ ( NoStop)

53. R. Laha, P. Lu, V. Takhistov, Gas heating from spinning and nonspinning evaporating primordial black holes. Phys. Lett. B 820, 136459 (2021). arXiv:2009.11837 [astro-ph.CO] (NoStop)

54. P. Lu, Sterile neutrinos and primordial black holes as dark matter candidates, Ph.D. thesis, school UCLA, Los Angeles (main) (2021) (NoStop)

55. S. Mittal, A. Ray, G. Kulkarni, B. Dasgupta, Constraining primordial black holes as dark matter using the global $21-\mathrm{cm}$ signal with Xray heating and excess radio background (2021). arXiv:2107.02190 [astro-ph.CO] (NoStop)

56. P.K. Natwariya, A.C. Nayak, T. Srivastava, Constraining spinning primordial black holes with global $21 \mathrm{~cm}$ signal (2021). arXiv:2107.12358 [astro-ph.CO] (NoStop)

57. Y. Luo, C. Chen, M. Kusakabe, T. Kajino, Impacts of Hawking radiation from primordial black holes in critical collapse model on the light element abundances. JCAP 05, 042 (2020). arXiv:2011.10937 [astro-ph.CO] (NoStop)

58. J.F. Acevedo, J. Bramante, A. Goodman, J. Kopp, T. Opferkuch, Dark matter, destroyer of worlds: neutrino, thermal, and existential signatures from black holes in the Sun and Earth. JCAP 04, 026 (2020). arXiv:2012.09176 [hep-ph] (NoStop) 
59. R. Calabrese, M. Chianese, D.F.G. Fiorillo, N. Saviano, Direct detection of light dark matter from evaporating primordial black holes (2021). arXiv:2107.13001 [hep-ph] (NoStop)

60. F. Schiavone, D. Montanino, A. Mirizzi, F. Capozzi, Axion-like particles from primordial black holes shining through the Universe (2021). arXiv:2107.03420 [hep-ph] (NoStop)

61. A. Arbey, J. Auffinger, M. Geiller, E.R. Livine, F. Sartini, Hawking radiation by spherically-symmetric static black holes for all spins: Teukolsky equations and potentials. Phys. Rev. D 103, 104010 (2021). arXiv:2101.02951 [gr-qc] (NoStop)

62. A. Arbey, J. Auffinger, M. Geiller, E.R. Livine, F. Sartini, Hawking radiation by spherically-symmetric static black holes for all spins: II - Numerical emission rates, analytical limits and new constraints (2021). arXiv:2107.03293 [gr-qc] (NoStop)

63. A. Cheek, L. Heurtier, Y.F. Perez-Gonzalez, J. Turner, Primordial black hole evaporation and dark matter production: I. Solely Hawking radiation (2021). arXiv:2107.00013 [hep-ph] (NoStop)

64. A. Cheek, L. Heurtier, Y.F. Perez-Gonzalez, J. Turner, Primordial black hole evaporation and dark matter production: II. Interplay with the freeze-in/out mechanism (2021). arXiv:2107.00016 [hepph] (NoStop)

65. G.F. Torres DelCastillo, Spin $3 / 2$ perturbations of algebraically special solutions of the Einstein-Maxwell equations. J. Math. Phys. 30, 2114 (1989) (NoStop)

66. G.F. Torresdel Castillo, G. Silva-Ortigoza, Rarita-Schwinger fields in the Kerr geometry. Phys. Rev. D 42, 4082 (1990) (NoStop)

67. G.F. Torresdel Castillo, G. Silva-Ortigoza, Spin $3 / 2$ perturbations of the Kerr-Newman solution. Phys. Rev. D 46, 5395 (1992) (NoStop)

68. D.N. Page, Particle emission rates from a Black Hole. 3. Charged Leptons from a Nonrotating Hole. Phys. Rev. D 16, 2402 (1977) (NoStop)

69. S. Kováčik, Hawking-radiation recoil of microscopic black holes (2021). arXiv:2102.06517 [gr-qc] (NoStop)

70. S. Hossenfelder, M. Bleicher, S. Hofmann, H. Stoecker, A.V. Kotwal, Black hole relics in large extra dimensions. Phys. Lett. B 566, 233 (2003). arXiv:hep-ph/0302247 (NoStop)

71. F. Moulin, A. Barrau, K. Martineau, An overview of quasinormal modes in modified and extended gravity. Universe 5, 202 (2019). arXiv:1908.06311 [gr-qc] (NoStop)

72. C.M. Harris, P. Kanti, Hawking radiation from a (4+n)-dimensional black hole: exact results for the Schwarzschild phase. JHEP 10, 014 (2003). arXiv:hep-ph/0309054 (NoStop)

73. A. Rincón, V. Santos, Greybody factor and quasinormal modes of Regular Black Holes. Eur. Phys. J. C 80, 910 (2020). arXiv:2009.04386 [gr-qc] (NoStop)
74. T. Berry, A. Simpson, M. Visser, General class of "quantum deformed" regular black holes (2021). arXiv:2102.02471 [gr-qc] (NoStop)

75. X.-C. Cai, Y.-G. Miao, Quasinormal modes and shadows of a new family of Ayón-Beato-García black holes. Phys. Rev. D 103, 124050 (2021). arXiv:2104.09725 [gr-qc] (NoStop)

76. O. Baake, C. Charmousis, M. Hassaine, M. SanJuan, Regular black holes and gravitational particle-like solutions in generic DHOST theories. JCAP 06, 021 (2021). arXiv:2104.08221 [hepth] (NoStop)

77. M. Molina and J.R. Villanueva, On the thermodynamics of the Hayward black hole. Class. Quantum Gravity 38, 105002 (2021). arXiv:2101.07917 [gr-qc] (NoStop)

78. E. Alesci, L. Modesto, Particle creation by loop black holes. Gen. Relativ. Gravity 46, 1656 (2014). arXiv:1101.5792 [gr-qc] (NoStop)

79. E. Alesci, L. Modesto, Hawking radiation from loop black holes. J. Phys. Conf. Ser. 360, 012036 (2012) (NoStop)

80. S. Hossenfelder, L. Modesto, I. Premont-Schwarz, Emission spectra of self-dual black holes (2012). arXiv:1202.0412 [gr-qc] (NoStop)

81. F. Moulin, K. Martineau, J. Grain, A. Barrau, Quantum fields in the background spacetime of a polymeric loop black hole. Class. Quantum Gravity 36, 125003 (2019). arXiv:1808.00207 [gr-qc] (NoStop)

82. M.A. Anacleto, F.A. Brito, J.A.V. Campos, E. Passos, Absorption and scattering by a self-dual black hole. Gen. Relativ. Gravity $\mathbf{5 2}$, 100 (2020). arXiv:2002.12090 [hep-th] (NoStop)

83. T. Sjöstrand, S. Ask, J.R. Christiansen, R. Corke, N. Desai, P. Ilten, S. Mrenna, S. Prestel, C.O. Rasmussen, P.Z. Skands, An introduction to PYTHIA 8.2. Comput. Phys. Commun. 191, 159 (2015). arXiv:1410.3012 [hep-ph] (NoStop)

84. J. Bellm et al., Herwig 7.2 release note. Eur. Phys. J. C 80, 452 (2020). arXiv:1912.06509 [hep-ph] (NoStop)

85. A. Coogan, L. Morrison, S. Profumo, Hazma: a Python toolkit for studying indirect detection of sub-GeV dark matter. JCAP 01, 056 (2019). arXiv:1907.11846 [hep-ph] (NoStop)

86. J. Chen, T. Han, B. Tweedie, Electroweak Splitting Functions and High Energy Showering, JHEP 11, 093 (2016). arXiv:1611.00788 [hep-ph] (NoStop) 